\title{
Distanciamento social e condições de vida na pandemia COVID-19 em Salvador-Bahia, Brasil
}

\author{
Social distancing and living conditions in the pandemic COVID-19 \\ in Salvador-Bahia, Brazil
}

\author{
Marcio dos Santos Natividade (https://orcid.org/0000-0002-3986-5656) ${ }^{1,2}$ \\ Kionna Bernardes (https://orcid.org/0000-0003-3181-2696) 2,3 \\ Marcos Pereira (https://orcid.org/0000-0003-3766-2502) 1,2 \\ Samilly Silva Miranda (https://orcid.org/0000-0002-1488-1246) ${ }^{1}$ \\ Juracy Bertoldo (https://orcid.org/0000-0003-3589-2494) 2,4 \\ Maria da Glória Teixeira (https://orcid.org/0000-0003-3318-3408) 1,2 \\ Humberto Lago Livramento (https://orcid.org/0000-0003-2366-7871) ${ }^{4}$ \\ Erika Aragão (https://orcid.org/0000-0002-4903-0556) 1,2
}

${ }^{1}$ Instituto de Saúde Coletiva, Universidade Federal da Bahia (UFBA). R. Basílio da Gama s/no, Canela. 40110040 Salvador BA Brasil. marcio.natividade@ outlook.com

${ }^{2}$ Rede CoVida: Ciências, Informação e Solidariedade. Salvador BA Brasil. ${ }^{3}$ Faculdade de Medicina, UFBA. Salvador BA Brasil.

${ }^{4}$ Programa Integrado Economia, Tecnologia e Inovação em Saúde, UFBA. Salvador BA Brasil.

\begin{abstract}
In the current scenario of the COVID-19 pandemic, Brazilian states and municipalities have adopted social distancing measures as a strategy to reduce the number of cases and control the disease. These measures affect populations and territories differently. This study aims to analyze the trend of social distancing in this pandem$i c$ and its relationship with the context of living conditions in Salvador, Bahia, Brazil. An ecological study with spatial distribution was conducted. The municipality's Social Distancing Index and the Living Conditions Index were calculated. Global and Local Moran Indices were employed to assess the degree of spatial dependence and autocorrelation. Fluctuations were observed in the social distancing levels during the analyzed period, with higher distancing percentages in neighborhoods with more favorable living conditions. The analysis and interpretation of COVID-19 containment measures, such as social distancing, should consider the profile of local vulnerability of each territory for the correct dimensioning of pandemic mitigation strategies from the perspective of developing social actions enabling greater adherence of the most impoverished populations. Key words Social distancing, Inequalities, Socioeconomic factors, Vulnerability study, Coronavirus infections
\end{abstract}

Resumo Na pandemia COVID-19, estados e municípios brasileiros adotaram medidas de distanciamento social como estratégia para reduziro número de casos e o controle da doença. Estas medidas atingem de forma diferenciada estratos populacionais e território. O objetivo deste estudo foi analisar a evolução do distanciamento social adotadas para o controle da pandemia COVID-19e sua relação com as condições de vida da população do município de Salvador, Bahia. Foi conduzido um estudo ecológico utilizando agregados espaciais. Calculou-se o Índice de Isolamento Social do município e o Índice de Condição de Vida. O Indice de Moran Global e Local foram usados para avaliar o grau de dependência e autocorrelação espacial. Observou-se oscilações nos indices de isolamento social durante o período analisado, com maiores percentuais de isolamento nos bairros com condições de vida mais favoráveis. A análise e a interpretação das medidas de contenção da COVID-19, a exemplo do distanciamento social, deve considerar o perfil de vulnerabilidade de cada território visando a monitorar o correto dimensionamento das estratégias de mitigação da pandemia, na perspectiva de desenvolver ações sociais capazes de possibilitar maior adesão das populações mais desfavorecidas.

Palavras-chave Isolamento social, Desigualdades, Fatores socioeconômicos, Estudo sobre vulnerabilidade, Infecções por coronavírus 


\section{Introdução}

O distanciamento social, entendido como uma estratégia não farmacológica que abrange o isolamento de casos, a quarentena dos contatos, e a prática voluntária de não frequentar ambientes com aglomerações de pessoas ${ }^{1}$, tem demonstrado alguma efetividade no controle do crescimento exponencial da doença. Essa estratégia pode resguardar os sistemas de saúde de um colapso devido à uma demanda muito maior do que a oferta, especialmente quando se trata de leitos de terapia intensiva ${ }^{2}$. Por outro lado, os impactos e os determinantes sociais e econômicos são discutidos como fatores limitantes desta estratégia, gerando discussões em torno do tempo de duração destas medidas de restrição ${ }^{3}$.

Em decorrência da disseminação do vírus SARS-Cov-2 em todo o mundo, e seguindo ações adotadas em outros países que tiveram sucesso no controle da pandemia, diversos estados e municípios brasileiros adotaram medidas de distanciamento social com o objetivo de diminuir o contato entre as pessoas e, consequentemente, controlar a velocidade da transmissão do vírus. Dentre estas medidas estão o cancelamento de eventos públicos, fechamento de escolas e empresas, recomendações para que as pessoas permaneçam em suas casas, dentre outras.

A diversidade do contexto socioeconômico e de desenvolvimento humano em âmbito regional foi uma das razões que estimulou a criação de um Comitê Científico da região Nordeste, composto por pesquisadores de referência nacional, tendo como objetivo propor e articular estratégias de combate e mitigação da pandemia de COVID-19. Dentre o conjunto de ações vinculadas a este comitê, destacam-se iniciativas de monitoramento das curvas epidêmicas de cada estado e de distanciamento social, com vistas à redução da demanda de internações hospitalares e números de óbitos.

Uma das formas de observar a efetividade do distanciamento social é por meio do Índice de Isolamento Social (IIS). Apesar de teoricamente o isolamento consistir na separação das pessoas doentes daquelas não infectadas ${ }^{4}$, este índice foi desenvolvido pela empresa Inloco para calcular o percentual da população que está respeitando as recomendações de isolamento, aqui entendida como medidas de distanciamento social. A Inloco é uma startup no ramo de tecnologia que tem acompanhado a movimentação de aproximadamente 60 milhões de brasileiros por meio da geolocalização de smartphones ${ }^{5}$.
No Brasil, o maior IIS foi registrado em 22 de março de 2020 (62,2\%), e em 09 de junho o país apresentou IIS de $38,2 \%$. Na região Nordeste, todos os estados possuíam neste mesmo período, índices abaixo de $60 \%$. A redução de contatos acima deste percentual tem o potencial de reduzir a transmissão da doença, quando associado a medidas de contenção (isolamento, quarenta de contato $)^{4}$. Na Bahia, o índice chegou a 45,7\% no dia 26 de maio, sendo considerado o quinto melhor do Brasil. A capital baiana, por sua vez, apresenta o terceiro melhor índice dentre todas as capitais do país, perdendo apenas para Macapá e Recife, respectivamente ${ }^{5,6}$.

Apesar da capital baiana apresentar bom índice de isolamento quando comparado com outras capitais do Brasil, tem-se observado uma elevação na ocorrência de casos e óbitos, revelando o crescimento ainda ascendente da doença ${ }^{7}$. Ao compreender que fatores econômicos e ambientais interferem diretamente no tempo em que as pessoas conseguem se manter em isolamento durante a pandemia ${ }^{8}$, é fundamental avaliar como o isolamento social se distribui entre os diferentes bairros da capital baiana, relacionando-o com as condições de vida da população. Assim, o objetivo deste estudo é analisar a evolução do distanciamento social adotadas para o controle da pandemia COVID-19 e sua relação com as condições de vida no município de Salvador.

\section{Metodologia}

Foi realizado um estudo ecológico de agregados espaciais em Salvador, Bahia, no período de 23 de março até 27 de abril de 2020 . A capital possui população estimada em 2019 de 2.872 .347 habitantes, com área equivalente à $693,453 \mathrm{~km}^{2}$ e densidade demográfica de $3.859,44 \mathrm{hab} . / \mathrm{km}^{2}{ }^{9}$.

A pesquisa analisou o distanciamento social e os aspectos relacionados à condição de vida entre bairros do município. A avaliação desta medida não farmacológica foi conduzida a partir do cálculo do IIS, que utilizou os dados de smartphones disponibilizados pela Inloco (registros de $1 \mathrm{mi}$ lhão de smartphones geolocalizados) $)^{5}$. Os dados foram obtidos considerando os deslocamentos de smartphone, sendo estes observados quando ocorreram fora de um raio de 400 metros das residências dos indivíduos. O IIS foi disponibilizado pela Inloco de forma agregada na unidade espacial em hexágonos e em seguida foi compatibilizado na unidade de bairros.

Considerou-se como proxy de condição de vida (CV) o Urban Health Index - UHI. Este é 
um índice que possibilita uma abordagem flexível para seleção, junção e apresentação de dados, tendo como finalidade prover de forma gráfica, visual e estatística as desigualdades em saúde ${ }^{10}$. O UHI compreende 7 indicadores sociodemográficos, calculado com base nos dados do censo demográfico do Brasil de 2010: 1- Proporção de residentes com renda domiciliar mensal per capita de até $1 / 2$ salário mínimo; 2- Proporção de pessoas negras; 3- Taxa de analfabetismo (proporção de pessoas com 15 e mais anos de idade analfabetas); 4- Taxa de escolaridade (proporção de pessoas com $15 \mathrm{e}$ mais anos de estudo); 5- Proporção de domicílios ligados a rede de abastecimento de água regular; 6- Proporção de domicílios com coleta de lixo regular; 7- Densidade domiciliar (número médio de pessoas por domicílio $)^{10}$. Esses indicadores receberam uma pontuação, que quando somadas resultaram em um escore para cada bairro referente ao ICV. Escores mais baixos de ICV corresponderam às piores condições de vida ${ }^{10}$.

Tendo em vista que parcela da população brasileira, assim como da capital baiana, encontra-se em contexto de vulnerabilidade social, foi necessário analisar também os indicadores de vulnerabilidade. Estes dimensionam aspectos estruturais de um determinado grupo populacional, condições de desenvolvimento humano e capacidade de resposta a um problema de saúde. O Instituto Votorantim desenvolveu o Índice de Vulnerabilidade Municipal (IVM) com o objetivo de identificar o grau de vulnerabilidade de cada município brasileiro em relação aos impactos provocados pela pandemia de COVID-19 ${ }^{11}$. O IVM utiliza dados secundários públicos obtidos em fontes oficiais e é composto por dimensões com diferentes pesos de composição, a saber: população vulnerável (32,35\%); economia local (11,76\%); estrutura dos serviços de saúde (23,53\%); organização do sistema de saúde (20,59\%); e capacidade administrativa $(11,76 \%)$. O escore final varia de 0 a 100 pontos e indica que, quanto maior o valor do IVM de um determinado município, mais vulnerável e suscetível ele está com relação aos impactos da COVID-19 $19^{11}$.

Para analisar o grau de dependência espacial entre os bairros em relação ao ISS foi calculado o Índice de Moran Global e Local. Esse índice varia de 0 a 1 , e quanto maior seu valor, mais dependente espacialmente.

Para a construção dos mapas temáticos foi utilizado o software QGIS versão 2.18 (Environmental Systems Research Institute - ESRI, Redlands, CA, Estados Unidos). Os cálculos de autocorrelação Global e Local foram realizados no software Geoda versão 2.14. Para gerenciamento das bases e outras análises foram utilizados o software R e Excel 2016.

\section{Resultados}

Observa-se oscilações nos IIS durante o período analisado no município de Salvador. Em 17 de março de 2020 o IIS foi de $26,0 \%$, alcançando maior percentual em 22 de abril $(67,0 \%)$. A partir dessa data os valores variaram, chegando a 50,1\%, no dia 27 deste mesmo mês. De forma irregular este indicador apresentou crescimento sendo a média entre 02 de fevereiro e 16 de março de 2020 de $22,5 \%$ e após esse período (17 de março a 27 de abril) de 51,4\% (Figura 1).

Quando da análise espacial do IIS, no período de 23 de março até 27 de abril de 2020, entre bairros do município estudado, foi possível observar uma distribuição irregular da adesão às medidas de distanciamento social (Figura 2). Os bairros com melhor adesão ao ISS foram em Stella Maris, Graça, Vitória, Barra, Garcia e Pituba, com média de $57,0 \%$. Em contrapartida, os bairros Jardim Cajazeiras, Jardim Santo Inácio, Pau da Lima, São Marcos, Arenoso e Sussuarana apresentaram as menores ocorrência de ISS, com registro médio de $48,0 \%$ no período analisado.

A análise do Índice de Moran Global e Local revelou alta correlação (I'moran global de 0,676, significante) nas áreas com maior adesão e baixa correlação nos bairros com menor adesão ao isolamento (Figura 3). Os maiores percentuais de isolamento concentraram-se em bairros com condições de vida mais favoráveis, especialmente aqueles localizados na orla marítima da cidade de Salvador (Figura 4).

A análise do IVM de Salvador aponta para um escore global de 47,7. As dimensões avaliadas pelo IVM indicam que o município apresenta escore de 45,7 para população vulnerável aos efeitos da COVID-19, destaque para o percentual de população idosa com $12,0 \%$; a população inscrita no Cadastro Único $(26,9 \%)$ e o número de internações por comorbidades associadas ao desenvolvimento de casos graves de COVID-19 por mil habitantes ${ }^{8,9}$. A dimensão com maior escore foi a de economia local com 65,8 . Este índice revela um PIB per capita de R $\$ 21.231,48$, com $29,4 \%$ da população empregada e com média de renda de 3,4 salários mínimos mensais para os trabalhadores formais.

A estrutura do sistema de saúde recebeu aproximadamente 46,0 pontos, composto pelo 


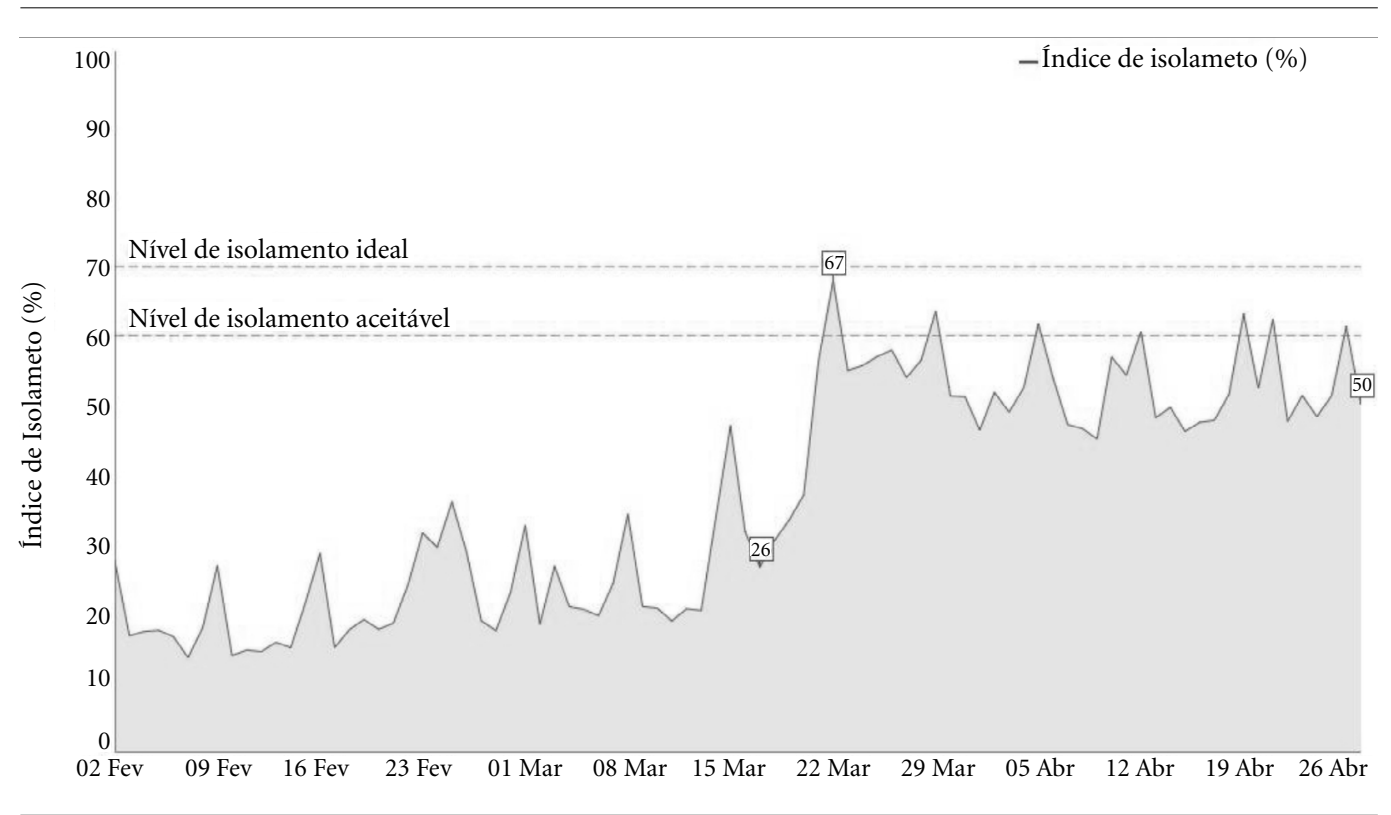

Figura 1. Evolução temporal do Índice de Isolamento Social no município de Salvador-BA, no período de 02/02/2020 a 27/04/2020.

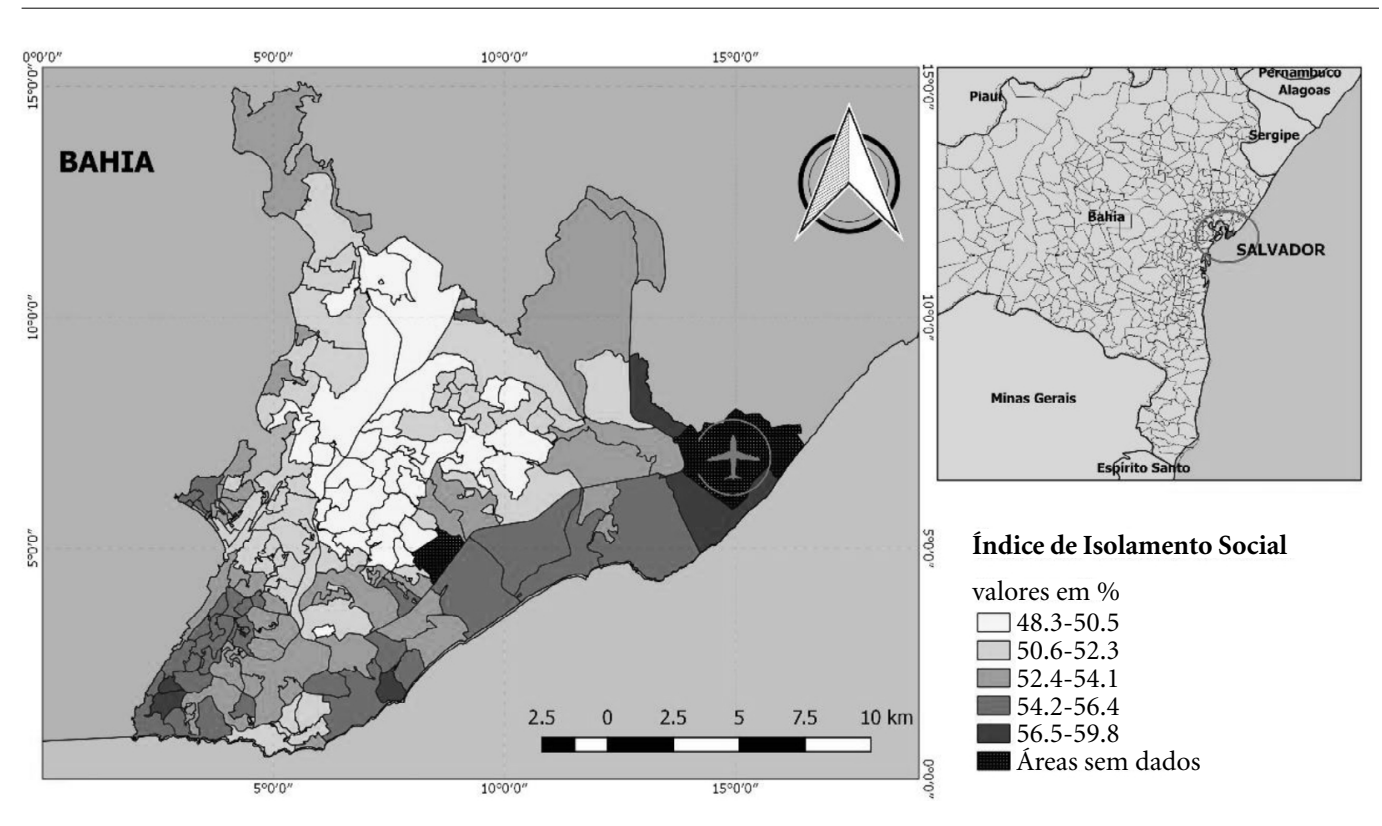

Figura 2. Distribuição espacial do Índice de Isolamento Social no município de Salvador-BA, no período de 23/03/20 a 27/04/20.

número de leitos hospitalares (24,6/10 mil hab.), número de leitos de UTI (25,4/100 mil hab.) e número de ventiladores e respiradores (44,7/100 mil hab.) na microrregião. Na organização dos serviços de saúde o escore foi de 54,0 , conside- rando que $71,8 \%$ da população é dependente do sistema público de saúde, sendo $37,9 \%$ referente a cobertura da atenção básica. A melhor pontuação do município foi na capacidade administrativa, com valor de 27,8 . 

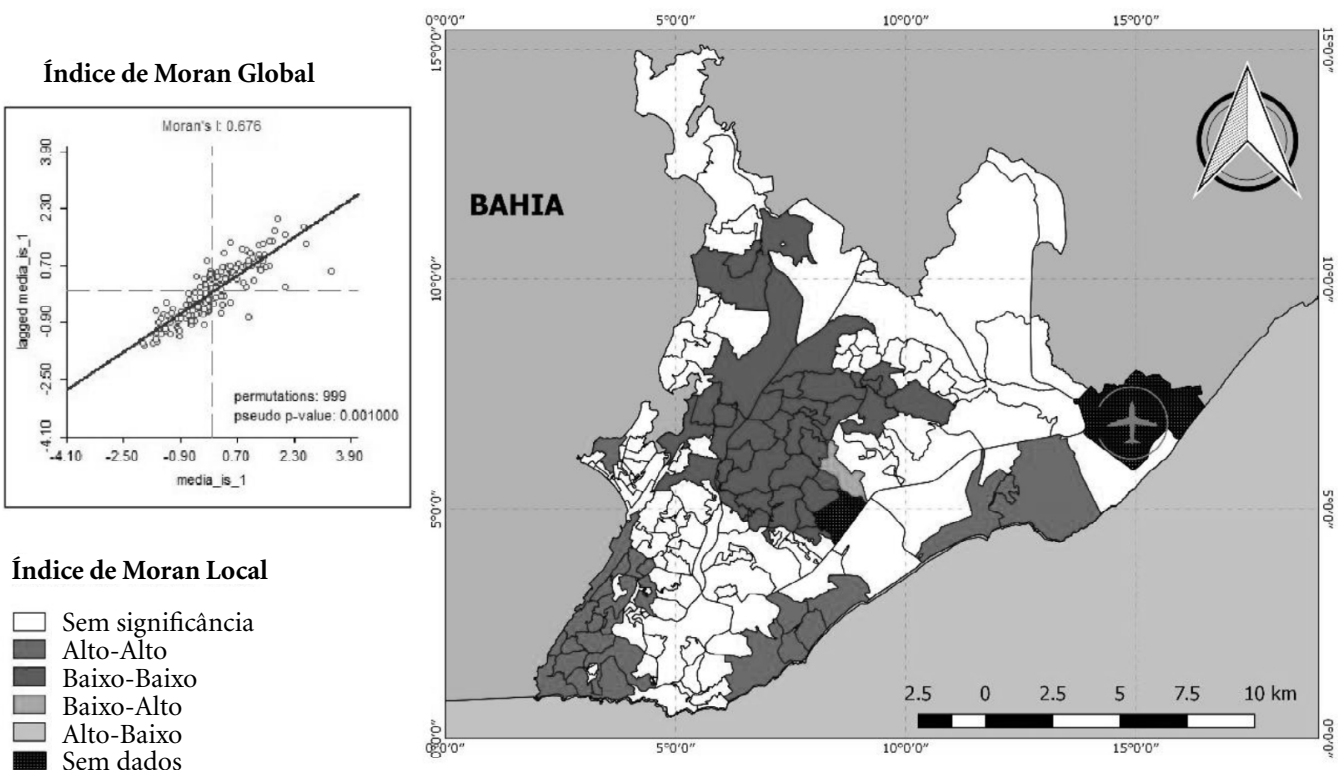

Índice de Moran Local

$\square$ Sem significância
Alto-Alto
$\square$ Baixo-Baixo
$\square$ Baixo-Alto
$\square$ Alto-Baixo
Sem dados

Figura 3. Padrão de autocorrelação espacial do Índice de Isolamento Social nos bairros do município de Salvador-BA, no período de 23/03/20 a 27/04/20.
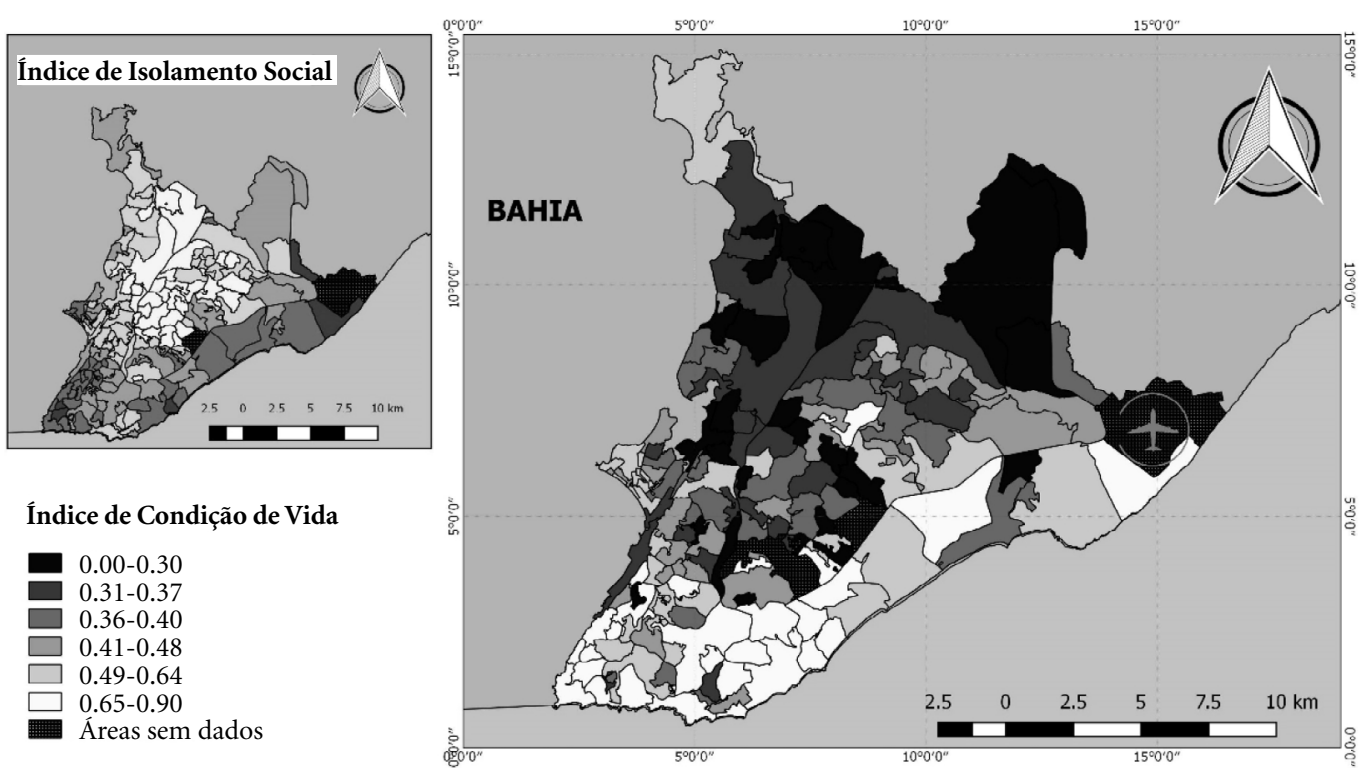

Índice de Condição de Vida

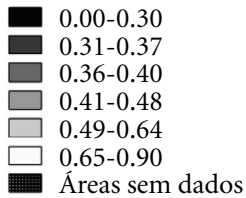

Figura 4. Distribuição espacial das Condições de Vida nos bairros do município de Salvador-BA, no período de $23 / 03 / 20$ a $27 / 04 / 20$.

\section{Discussão}

Este estudo sinaliza uma possível relação entre isolamento social e condições de vida em Salvador-Bahia. Os bairros considerados com maior índice de pobreza ${ }^{12}$ são aqueles que possuem menor percentual do IIS.

Ademais, o município apresenta índice de vulnerabilidade alto, que o coloca em desvantagem para o enfrentamento da pandemia, quando 
comparado com outras capitais do Brasil, como São Paulo (escore global 41,2), representado em dimensões específicas de população vulnerável com escore de 55,4, aspectos de economia local $(34,5)$, além da estrutura dos serviços de saúde $(40,1)$, organização dos serviços de saúde com melhor pontuação 26,1 e capacidade de administração municipal $(39,9)$. Outro município utilizado como referência foi Curitiba (escore global 45,9 ), composto por escores de população vulnerável de 50,3, economia local de 34,6, estrutura e organização dos serviços de saúde com escores de 46,5 e 42,5, respectivamente, além de capacidade administrativa com $45,9^{11}$.

Os resultados apontam para um quadro de grandes desigualdades sociais e concentração de renda, revelando que a capital baiana continua segregada por diferentes condições sociais. As camadas de alta e média renda estão concentradas na área central ou na orla marítima, enquanto os grupos de menor renda se aglomeram principalmente em áreas periféricas ou nas fronteiras com os municípios circunvizinhos ${ }^{13}$.

A baixa adesão às medidas de distanciamento social pode ser determinada pela desigualdade social. Isso ocorre porque a distribuição da saúde e da doença em populações não é aleatória, estando associada à posição social, que por sua vez define as condições de vida e de trabalho de populações ${ }^{14}$. Por outro lado, deve-se considerar que o distanciamento social pode ser influenciado por aspectos culturais, de características dos sistemas políticos e de saúde, bem como dos procedimentos operacionais para implementar estratégias de mitigação da pandemia ${ }^{4}$.

Neste contexto, observa-se que alguns fatores podem estar associados ao comportamento da população durante o isolamento social. Aspectos relacionados à ocupação e medo do desemprego são questões a serem consideradas. Parcelas importantes da população que sobrevivem de trabalho no mercado informal encontram dificuldades na adesão ao isolamento, pois necessitam complementar renda, ainda que estejam em programa temporário de transferência de renda ${ }^{3,8,15}$. Ademais, o contexto de trabalho informal limita as condições de proteção ao trabalhador, com maior exposição a riscos e necessidade de manutenção da atividade informal como meio de sobrevivência. A qualidade da informação, a credibilidade dos governantes e a incerteza sobre o vírus também repercutem na adesão. A maneira como as autoridades de saúde pública reportam sobre a importância do isolamento podem trazer maior ou menor confiança na medida ${ }^{8,15}$.
Ressalta-se ainda, que pessoas com menor renda, além de mais expostas aos impactos financeiros ocasionados pela pandemia da COVID-19, também estão mais vulneráveis a serem afetadas por problemas de saúde física e psicológicos associados a reclusão necessária no período de isolamento social ${ }^{8,15}$. Tais condições de vulnerabilidade podem ser mais danosas nas comunidades formadas por famílias que vivem em único cômodo doméstico, pois a possibilidade de contaminação pela COVID-19 pode ser maior ${ }^{3,8,15}$. Gera-se, portanto, a necessidade de se superar os desafios em torno das medidas efetivas para a promoção do isolamento social.

Diante desse cenário de iniquidades no município de Salvador e dada a importância das medidas não farmacológicas para redução do número de casos de COVID-19, única estratégia de controle da doença por não se dispor de nenhuma terapia específica nem de vacinas ${ }^{16}$, cumpre alertar da necessidade de estimular e criar condições reais, por meio de políticas públicas emergenciais, para ampliar a adesão da população ao distanciamento social, principalmente nas áreas com maior desigualdade. Sem distanciamento social significativo ou uma combinação de distanciamento social moderado aliado à busca ativa de casos para se proceder ao necessário isolamento, a progressão exponencial da epidemia é inexorável e os serviços de saúde entrarão em colapso ${ }^{17}$.

$\mathrm{O}$ isolamento de casos e contatos associados ao distanciamento social, além de diminuir a incidência da doença, têm um impacto econômico considerável. Wang et al. ${ }^{18}$ revelaram um impacto positivo do ponto de vista econômico, ao analisar o contexto chinês. $\mathrm{O}$ isolamento e a quarentena constituíram as intervenções mais econômicas, evitando 1.696 casos e economizando em torno de US\$ 11.515 com gastos de saúde ${ }^{18}$.

Embora as estratégias não farmacológicas tenham impactos positivos, principalmente no que se refere à capacidade de salvar vidas ${ }^{19}$, um colapso econômico também se constitui em risco importante para o desenvolvimento de problemas de saúde. Eventualmente, a pandemia afetará economicamente todos, e uma recessão global é iminente ${ }^{20}$. A contenção da propagação e a redução do impacto econômico da pandemia exigirá ações coordenadas entre os setores da economia e da saúde para mitigar os problemas decorrentes destas duas crises ${ }^{21}$.

A adesão ao isolamento, por exemplo, depende além de outras questões, de medidas de proteção social que garantam recursos para a população economicamente mais vulnerável. No caso 
do Brasil, o auxílio emergencial previsto na Lei $\mathrm{n}^{\circ}$ $13.982 / 2020$, modificada com texto substitutivo do PL 873/2020, que amplia o auxílio emergencial de $\mathrm{R} \$ 600,00$ a mais categorias de pessoas em condições vulneráveis é considerado insuficiente. De acordo com informações disponibilizadas no site do senado, mais de 96 milhões já solicitaram este auxílio emergencial, e 50,5 milhões de pessoas foram consideradas elegíveis, o que evidenciou o expressivo contingente da população brasileira que vive em condições precárias ${ }^{22}$.

Em suma, as condições de vida de parcelas consideráveis da população de Salvador impõem claras limitações ao distanciamento social e à adoção das medidas de higiene, apontadas pelas organizações sanitárias como essenciais para evitar a contaminação pelo vírus, tendo em vista que esta cidade é a capital de um estado situado em uma das regiões mais pobres do país. As dificuldades inerentes à situação em que se vive, requerem medidas complementares por parte dos governos, que garantam padrões mínimos de higiene, salubridade e bem-estar. As pessoas de baixa renda que residem nas áreas periféricas ou favelas estão sujeitas a uma série de riscos e ameaças à sua sobrevivência (acesso à alimentação e padrões mínimos de higiene e salubridade), bem como maior risco à integridade física e psicológica, com destaque para as mulheres que estão em sua maioria expostas à violência e à sobrecarga de trabalho doméstico ${ }^{23}$.

Apesar da limitação inerente a este estudo, já que dados de smartphones podem ter sido provenientes majoritariamente de pessoas de alta renda, os achados revelam que a análise das medidas de contenção como distanciamento social não deve ser feita de forma isolada e deve considerar o perfil de vulnerabilidade local para o correto dimensionamento das estratégias de prevenção e controle. O planejamento do município deve, portanto, basear-se no cenário epidemiológico, considerando as diferenças e as iniquidades entre diferentes bairros, sendo recomendável a implementação/manutenção de políticas de proteção social, e o desenvolvimento de ações que contribuam para a manutenção dos empregos e da renda dos trabalhadores, dando prioridade aos grupos mais vulnerabilizados. É imperativo estabelecer suporte financeiro, a ser distribuído de forma ágil e desburocratizada, pelos diferentes entes federados para garantir renda para os trabalhadores informais, mães chefes de família, dentre outros.

Por fim, é preciso destacar que apesar do distanciamento social no município analisado e seus respectivos bairros não terem alcançado os valores desejados, esta continua sendo uma das mais importantes medidas de controle e prevenção para a COVID-19. Em decorrência do número de casos confirmados, número de óbitos, quantidade de leitos e de testes, iniquidades sociais extremas, além do percentual de adesão ao distanciamento social abaixo do considerado ideal na capital baiana, entendemos que não é o momento de flexibilização, visto que muito ainda precisa ser desenvolvido para reduzir a transmissão e a produção de casos e, por conseguinte, controlar esta pandemia. Abordagens metodológicas complementares são necessárias para ampliar o entendimento das repercussões das condições de vida e de distanciamento social na ocorrência e gravidade da COVID-19 na população.

\section{Colaboradores}

MS Natividade concebeu o estudo, realizou a coleta, análise de dados e escrita do artigo. K Bernardes, M Pereira, SS Miranda e E Aragão contribuíram na escrita e revisão do artigo. J Bertoldo e HL Livramento contribuíram na análise dos dados e revisão crítica do artigo. MG Teixeira realizou a revisão crítica do estudo. Todos os autores aprovaram a versão final do estudo.

\section{Agradecimentos}

Os autores gostariam de agradecer a Luiza Botelho, Luciano Mello e Afonso Delgado da Inloco, pela valiosa colaboração com a disponibilidade dos dados sobre o Índice de Isolamento Social e as diversas consultas realizadas ao longo dessa publicação. 


\section{Referências}

1. Qualls N, Levitt A, Kanade N, Wright-Jegede N, Dopson S, Biggerstaff M, Reed C, Uzicanin A. Community mitigation guidelines to prevent pandemic influenza - United States, 2017. MMWR Recomm and Rep 2017; 66(1):1-34.

2. Ferguson NM, Laydon D, Nedjati-Gilani G, Imai N, Ainslie K, Baguelin M, Bhatia S, Boonyasiri A, Cucunubá Z, Cuomo-Dannenburg G, Dighe A, Dorigatti I, Fu H, Gaythorpe K, Green H, Hamlet A, Hinsley W, Okell LC, Elsland SV, Thompson H, Verity R, Volz E, Wang H, Wang Y, Walker PG, Walters C, Winskill P, Whittaker C, Donnelly CA, Riley S, Ghani AC. Impact of non-pharmaceutical interventions (NPIs) to reduce COVID-19 mortality and healthcare demand. Imperial Ac Uk 2020; 1-20.

3. Ahmed F, Ahmed N, Pissarides C, Stiglitz J. Why inequality could spread COVID-19. Lancet Public Health 2020; 5(5):e240.

4. Aquino EML, Silveira IH, Pescarini JM, Aquino R, Souza-Filho JA. Medidas de distanciamento social no controle da pandemia de COVID-19: potenciais impactos e desafios no Brasil. Cien Saude Colet 2020; 25(Supl. 1):2423-2446.

5. Inloco [Internet]. Geolocalização e privacidade: os dados no combate à COVID-19. [cited 2020 Jun 10]. Available from: https://www.inloco.com.br/covid-19.

6. Bahia. Secretaria da Saúde do Estado da Bahia (Sesab). Bahia está em $5^{\circ}$ lugar no ranking nacional em isolamento social [Internet]. [cited 2020 Jun 10]. Available from: http://www.saude.ba.gov.br/2020/05/27/ bahia-esta-em-5o-lugar-no-ranking-nacional-de-isolamento-social/

7. Rede Covid. PAINEL - Rede Covida [Internet]. [cited 2020 Jun 10]. Available from: https://painel.covid19br.org/

8. Bezerra ACV, Silva CEM, Soares FRG, Silva JAM. Fatores associados ao comportamento da população durante o isolamento social na pandemia de COVID-19. Cien Saude Colet 2020; 25(Supl. 1):2411-2421.

9. Instituto Brasileiro de Geografia e Estatística (IBGE). Cidades@: Bahia: Salvador: Panorama [Internet]. [cited 2020 Jun 10]. Available from: https://cidades.ibge. gov.br/brasil/ba/salvador/panorama

10. World Health Organization (WHO). The Urban Health Index: a handbook for its calculation and use [Internet]. 2014 [cited 2020 Jun 10]. Available from: www. who.int

11. Instituto Votorantim. Índice de Vulnerabilidade $\mathbf{M u -}$ nicipal - COVID-19 [Internet]. [cited 2020 Jun 10]. Available from: http://institutovotorantim.org.br/ municipioscontraocorona/ivm/

12. Santos EI, Barreto RCS, Carvalho ICS. A pobreza multidimensional em Salvador diminuiu? Evidências a partir da abordagem espacial. Contextus - Rev Contemporânea de Economia e Gestão 2018; 15(3):181201.

13. Carvalho IMM, Pereira GC. Salvador: Transformações na ordem urbana. Rio de Janeiro: Observatório das Metrópoles; 2014.
14. Buss PM, Pellegrini Filho A. A saúde e seus determinantes sociais. Physis 2007; 17(1):77-93.

15. Ölcer S, Yilmaz-Aslan Y, Brzoska P. Lay perspectives on social distancing and other official recommendations and regulations in the time of COVID-19: a qualitative study of social media posts. BMC Public Health 2020; 20(1):1-9.

16. World Health Organization Writing Group, Bell D, Nicoll A, Fukuda K, Horby P, Monto A, Hayden F, Wylks C, Sanders L, van Tam J. Nonpharmaceutical interventions for pandemic influenza, international measures. J Emerg Infect Dis 2006; 12(1):81-87.

17. Tuite AR, Fisman DN, Greer AL. Mathematical modelling of COVID-19 transmission and mitigation strategies in the population of Ontario, Canada. CMAJ 2020; 192(19):e497-505.

18. Wang Q, Shi N, Huang J, Cui T, Yang L, Ai J, Ji H, Xu $\mathrm{K}$, Ahmad T, Bao C, Jin $\mathrm{H}$. Effectiveness and cost-effectiveness of public health measures to control COVID-19: a modelling study. medRxiv 2020; 2020.03.20.20039644.

19. Nussbaumer-Streit B, Mayr V, Dobrescu AI, Chapman A, Persad E, Klerings I, Wagner G, Siebert U, Christof, Zachariah C, Gartlehner. Quarantine alone or in combination with other public health measures to control COVID-19: a rapid review. Cochrane Database Syst Rev 2020; (4):CD013574.

20. Khoo EJ, Lantos JD. Lessons learned from the COVID-19 pandemic. Acta Paediatr 2020; 109(7):13231325.

21. Ataguba JE. COVID-19 Pandemic, a War to be Won: Understanding its Economic Implications for Africa. Appl Health Econ Health Policy 2020; 18(3):325-328.

22. Auxílio emergencial: 96 milhões de pessoas solicitaram benefício pelo aplicativo, explica Onyx. TV Senado. [Internet]. [cited 2020 Jun 10]. Available from: https://www12.senado.leg.br/tv/programas/noticias-1/2020/05/96-milhoes-de-brasileiros-acessaram -aplicativo-para-solicitar-auxilio-emergencial-explica-onyx

23. Rocha R, Pires C. Nota Técnica Abril de 2020. Os efeitos sobre grupos sociais e territórios vulnerabilizados das medidas de enfrentamento à crise sanitária da COVID-19: propostas para o aperfeiçoamento da ação pública. [Internet]. [cited 2020 Jun 10]. Available from: https://www.ipea.gov.br/portal/images/stories/PDFs/ nota_tecnica/200408_nota_tenica_diest.pdf

Artigo apresentado em 10/06/2020

Aprovado em 29/06/2020

Versão final apresentada em 01/07/2020 